\title{
Interest of broadband optical monitoring for thin-film filter manufacturing
}

\author{
Bruno Badoil, Fabien Lemarchand, ${ }^{*}$ Michel Cathelinaud, and Michel Lequime \\ Institut Fresnel, Unité Mixte de Recherche 6133, Université Paul Cezanne, 13397 Marseille Cedex 20, France \\ ${ }^{*}$ Corresponding author: fabien.lemarchand@fresnel.fr
}

Received 7 November 2006; revised 8 March 2007; accepted 18 March 2007; posted 19 March 2007 (Doc. ID 76830); published 20 June 2007

\begin{abstract}
Broadband optical monitoring for thin-film filter manufacturing is more and more developed thanks to better performances of spectrometers with array detectors. We compare this optical monitoring with turning point monitoring and quartz monitoring of different designs. The sensitivity to thickness errors and to refractive index errors is evaluated. We show that real time determination of deposited thickness is a valuable criterion. We also present our experimental setup of transmittance and reflectance broadband optical monitoring. The use of a 400-1000 nm range combined with a signal-to-noise ratio of $\sim 2500$ in transmittance and 1000 in reflectance permits us to expect the manufacturing of high-performance non-quarter-wave designs. A first manufacturing of an 18-layer non-quarter-wave high-pass filter is provided. (C) 2007 Optical Society of America
\end{abstract}

OCIS codes: $310.0310,310.1860,310.1620,310.6860$.

\section{Introduction}

The success of the manufacturing of a thin-film filter is undoubtedly linked to a high-performance thickness monitoring system. Thickness control methods during deposition include nonoptical and optical methods [1,2]. From a practical point of view, nonoptical methods are generally easier to implement inside a vacuum deposition chamber. Among these methods quartz crystal monitoring is a widely developed method in the optical coating industry. Layer thickness measurement is performed by a sensor made of a thin metalized wafer of crystalline quartz. The quartz frequency shift measurement provides an estimation of the average evaporation rate and the film thickness. Concerning the thickness measurement, the most accepted advantages are the ease of installation, the possibility of installing several sensors that are simultaneously active, and the possibility of measuring very thin layers, in the $10 \mathrm{~nm}$ range. One of the drawbacks is that it is not a direct measurement of the film thickness. Moreover, the deposition of relatively thick stacks (in the $2-10 \mu \mathrm{m}$ range) can induce a change in the shape of the evapo-

0003-6935/07/204294-10\$15.00/0

(C) 2007 Optical Society of America rant stream and the choice of an indirect measurement, such as quartz monitoring, should be carefully studied. And finally, one must be aware of the eventual difference of temperature on the substrates and on the crystal sensor. In spite of all these disadvantages, the choice of quartz monitoring is widely spread in the optical coating industry and can provide very efficient components, such as antireflective coatings. But, for some of them, such as narrow bandpass filters, an optical monitoring is a more suitable solution. The optical monitoring (also named radiometric monitoring if the spectral range extends from the UV to the IR), consists in measuring in situ a light flux reflected or transmitted by a substrate located in the substrate holder. The analysis of this flux provides information about deposited material and more precisely about its optical thickness. One of the first experimental demonstrations was written about in [3] and several efficient commercial systems are available today [4]. One other optical method for the deposition control is in situ ellipsometry [5]. It relies on changes in the state of polarization that depends on the optical nature of the deposited film. Note that the several strategies based on optical monitoring can be edited. One can choose a direct control of the component itself, an indirect monitoring by performing measurements on a separate surface, or a composite 
monitoring using both strategies. For instance, an optical monitoring system working in transmission prohibits a direct monitoring if one of the layers is an opaque metallic, such as for metal-dielectric light absorbers [6].

An advantage of these optical monitoring systems is that the optical thickness $n \times t$ is controlled at a particular wavelength. Thus, even if a drift in the refractive index occurs during deposition, at least the correct $n \times t$ is obtained. From an industrial point of view, the main disadvantages of optical monitoring are the cost and the complexity of installation since, first, windows must be installed inside the vacuum deposition chamber and, second, the entire optical system requires enough space, a precise mechanical alignment, and a vibration-free environment. To complete this view of the optical monitoring systems, one should not ignore real time broadband optical monitoring (BOM) systems, which have been recently introduced and are already commercially available. The principle consists in a sequential measurement of reflectance and/or transmittance as a function of wavelength. The analysis of the spectrum provides information about the film thickness, the refractive index, and the layer absorption. Such systems, working in transmission, use spectrophotometers with array detectors [7-11]. Anti-reflection (AR) broadband [9] and ion-assisted deposition long-pass filter coatings [8] with good reproducibility were performed. The theoretical interest of $\mathrm{BOM}$ has been recently investigated in [12].

The aim of this paper is to make a comparison of the efficiency of three monitoring methods, including the most widely used one, quartz crystal thickness measurement, one of the most efficient, i.e., the classical photometric optical monitoring in transmission, and the most recent one, the BOM system. The reproducibility of the manufacturing of several designs (antireflective coating, bandpass filter, and beam splitter) will be theoretically tested, taking into account uncertainties about quartz or optical signals, or even errors in refractive indexes.

We will show that an appropriate strategy applied to BOM systems enables us to achieve quite good performances. The stopping criterion, dependant upon the spectral behavior of transmittance/reflectance, will be precisely defined. In the latter part, we will detail the implantation of a reflection and transmission BOM system on a thin-film deposition chamber. The first experimental result, concerning a long-pass filter with a cutoff wavelength of $700 \mathrm{~nm}$ will be given.

\section{Experimental Setup for an Optical Broadband Monitoring Method Using Both Transmittance and Reflectance}

\section{A. Description of the System}

The dedicated deposition machine is a Balzers BAK 800 with a high energetic deposition process. It uses reactive low-voltage ion plating (RLVIP) deposition method. The optical system developed is able to mea-

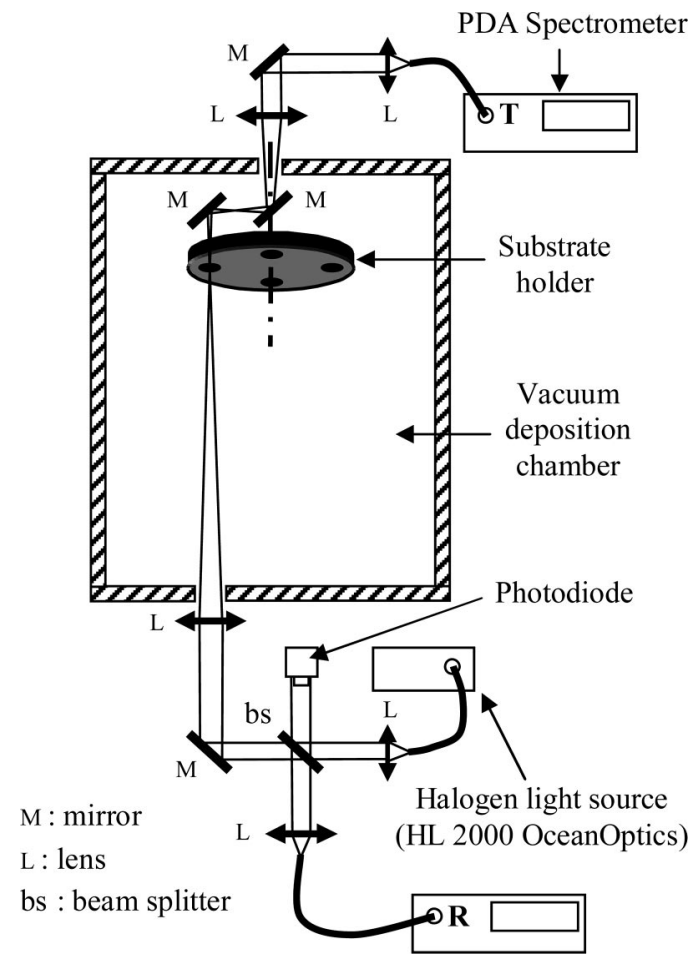

Fig. 1. Experimental setup of a BOM implanted on a Balzers BAK800 deposition chamber.

sure simultaneously both transmission and reflection by way of a two-channel array detector in the 400 $1000 \mathrm{~nm}$ range. Figure 1 gives a schematic of the whole system.

For more flexibility, the halogen light source and the spectrometer are connected to the system by optical fibers. Beneath the deposition chamber, the entrance fiber, which has a diameter of $200 \mu \mathrm{m}$, is imaged on the sample with a magnification ratio equal to 35 , corresponding to an analysis beam diameter of $7 \mathrm{~mm}$. A broadband beam splitter in the 400 $1000 \mathrm{~nm}$ range allows us to collect the reflected light on the sample through an optical fiber connected to one channel of the spectrometer. We notice that the beam splitter also permits us to collect a reference beam and enables us to balance fluctuations in the light source. This reference beam is measured by a silicon photodiode without any spectral analysis.

Similarly, the beam transmitted by the sample is collected above the deposition chamber and is focused on an optical fiber connected to the second channel of the spectrometer. Notice that, as the window transmitting this beam out of the chamber is centered on the rotation axis of the substrate holder, we use a two-mirror periscopic system located above the substrate holder. Finally, all of the optical images are measured with two-lens telecentric systems so that the angular distribution of the light is preserved and allows for an optimum efficiency between the entrance and exit fibers.

Concerning the choice of the spectrometer, the spectral range should at least extend from 400 to 
$1000 \mathrm{~nm}$ with a spectral resolution of $\sim 3 \mathrm{~nm}$. However, the most selective parameter for us was the signal-to-noise ratio (SNR), at least $1000 \mathrm{~nm}$, for a maximum integration time equal to $50 \mathrm{~ms}$ corresponding to the rotation of the substrate across the monitoring beam. Two major families of array detectors coexist: CCD and photodiode array (PDA). A CCD requires less of an electrical charge than a PDA and also has a higher charge-to-voltage conversion efficiency, making this technology ideal for low-lightlevel detection. A PDA, on the other hand, is more suited for applications where the light level is relatively high. Indeed, the photon saturation charge is greater for a PDA than for a CCD, so its dynamic range is wider. Our application concerns direct measurements of reflectance and transmittance. A CCD detector would require the use of a neutral density on the optical path in order to avoid saturation, leading to a SNR that is worse than for a PDA detector without using any density.

We finally selected a two-channel ZEISS MCS 501 UV-near-infrared (NIR) spectrometer with an electronic multiplexer and a 1024 photodiode array [13]. The spectrometer uses a 15 bit analog-to-digital converter, and presents a slight nonlinearity of the detector above 16,000 counts. This led us to choose this value as the maximum nondistorted signal.

Due to the rotation of the monitoring sample, measurements are performed every $2 \mathrm{~s}$. To calculate the transmittance and reflectance of the coating during its manufacturing, all measurements are normalized with the corresponding signals measured on a bare substrate before starting the deposition. The software we developed to perform data acquisition permits us to store all measured spectra for the postprocessing analysis, index characterization, for example. It also permits real-time signal processing to determine the moment to stop the layer deposition, based on any monochromatic, polychromatic, or broadband reflectance or transmittance criteria.

\section{B. Modeling of Measured Data and Performance of the System}

The performance of the system should be better in transmittance than in reflectance for at least two reasons. The first reason is linked to the nature of the bare substrate, which is generally made of glass. The initial transmitted flux is hence more than ten times higher than the reflected flux. Fluctuations in the reference signal are then more important for the reflectance channel. The second reason is linked to dynamic aspects. The rotation movement of the substrate holder induces instabilities in the plane of the substrate and, as a matter of fact, produces a deviation in the reflected beam, which may not entirely be injected into the fiber.

After several trials in which we used different models, we have chosen the following modeling of the experimental noise of our system: the transmittance and/or reflectance measurements (values from 0 to 1 ) are represented by Eqs. (1) and (2):

$$
\begin{aligned}
& T_{\text {meas }}(\lambda)=\alpha_{t} T_{\text {th }}(\lambda)+\beta_{t}(\lambda), \\
& R_{\text {meas }}(\lambda)=\alpha_{r} R_{\text {th }}(\lambda)+\beta_{r}(\lambda),
\end{aligned}
$$

where $T_{\text {meas }}$ and $R_{\text {meas }}$ stand for experimental signals at a given wavelength $\lambda, T_{\text {th }}$ and $R_{\text {th }}$ stand for the theoretical signals. Our model consists of associating $\alpha_{t}$ and $\alpha_{r}$ with a normal law with a mean equal to 1 (multiplicative noise) and a standard deviation, respectively, $\sigma_{\alpha t}$ and $\sigma_{\alpha r}$. $\beta_{t}$ and $\beta_{r}$ are assimilated to a normal law with zero mean (additive noise) and standard deviations of $\sigma_{\beta t}$ and $\sigma_{\beta r}$. One possible interpretation of this modeling consists of assimilating $\beta$ to an intrinsic static noise of the system (essentially due to the association light source-detector) and $\alpha$ to a specific noise partially due to the dynamic aspect of the measurement (rotation of the substrate holder). Analyzing $N$ sets of experimental measurements, we can deduce the distributions $\alpha^{(n)}$ and $\beta^{(n)}(\lambda)(1 \leq n$ $\leq N$ ) from the following equations:

$$
\begin{aligned}
\alpha^{(n)} & =\frac{\frac{1}{N_{\lambda}} \sum_{\lambda} S_{\text {meas }}^{(n)}(\lambda)}{\frac{1}{N_{\lambda}} \sum_{\lambda} S_{\text {th }}(\lambda)}, \\
\beta^{(n)}(\lambda) & =S_{\text {meas }}^{(n)}(\lambda)-\alpha^{(n)} S_{\text {th }}(\lambda),
\end{aligned}
$$

with

$$
S_{\mathrm{th}}(\lambda)=\frac{1}{N} \sum_{n=1}^{N} S_{\text {meas }}^{(n)}(\lambda),
$$

where $S_{\text {meas }}(\lambda)$ and $S_{\text {th }}(\lambda)$ respectively stand for the measurement and theoretical signals. $N_{\lambda}$ is the number of wavelengths.

As an illustration of the relevance of this modeling, we have plotted the scattering values of $\sigma_{\alpha}$ and $\sigma_{\beta}$ at different levels of transmitted and reflected flux in Fig. 2. $\sigma_{\beta}$ is determined at a particular wavelength of $\lambda=600 \mathrm{~nm}$, whereas $\sigma_{\alpha}$ is deduced from the fluctuations of the average value of the transmittance of the whole spectrum. For each level of transmittance and reflectance, 100 acquisitions have been performed to statistically determine the values of $\sigma_{\alpha}$ and $\sigma_{\beta}$. The relative flatness of the scattering points confirms the pertinent modeling of the experimental data. Figure 3 is an illustration of the dispersion of values of $\sigma_{\beta}$ with a wavelength. We have plotted the average values of $\sigma_{\beta}$ (considering variable values of transmittance) at different wavelengths $\lambda$ that vary from 400 to $1000 \mathrm{~nm}$. $\sigma_{\beta}$ is lower than $2 \times 10^{4}$ from 520 to $800 \mathrm{~nm}$. The increase of $\sigma_{\beta}$ below $500 \mathrm{~nm}$ is caused by the low-light flux of the halogen lamp whereas, above $800 \mathrm{~nm}$, the PDA detector is less sensitive. A more powerful light source may improve the spectral range of an optimal utilization of the BOM. Table 1 summarizes the different average values of variance in the 500-900 $\mathrm{nm}$ range over flux between 0.1 and 1 . If the two noises $\alpha$ and $\beta$ are independent, which is likely, the SNR for a maximum signal is given by

$$
\mathrm{S} / \mathrm{N}=\frac{1}{\sqrt{\sigma_{\alpha}^{2}+\sigma_{\beta}^{2}}} .
$$



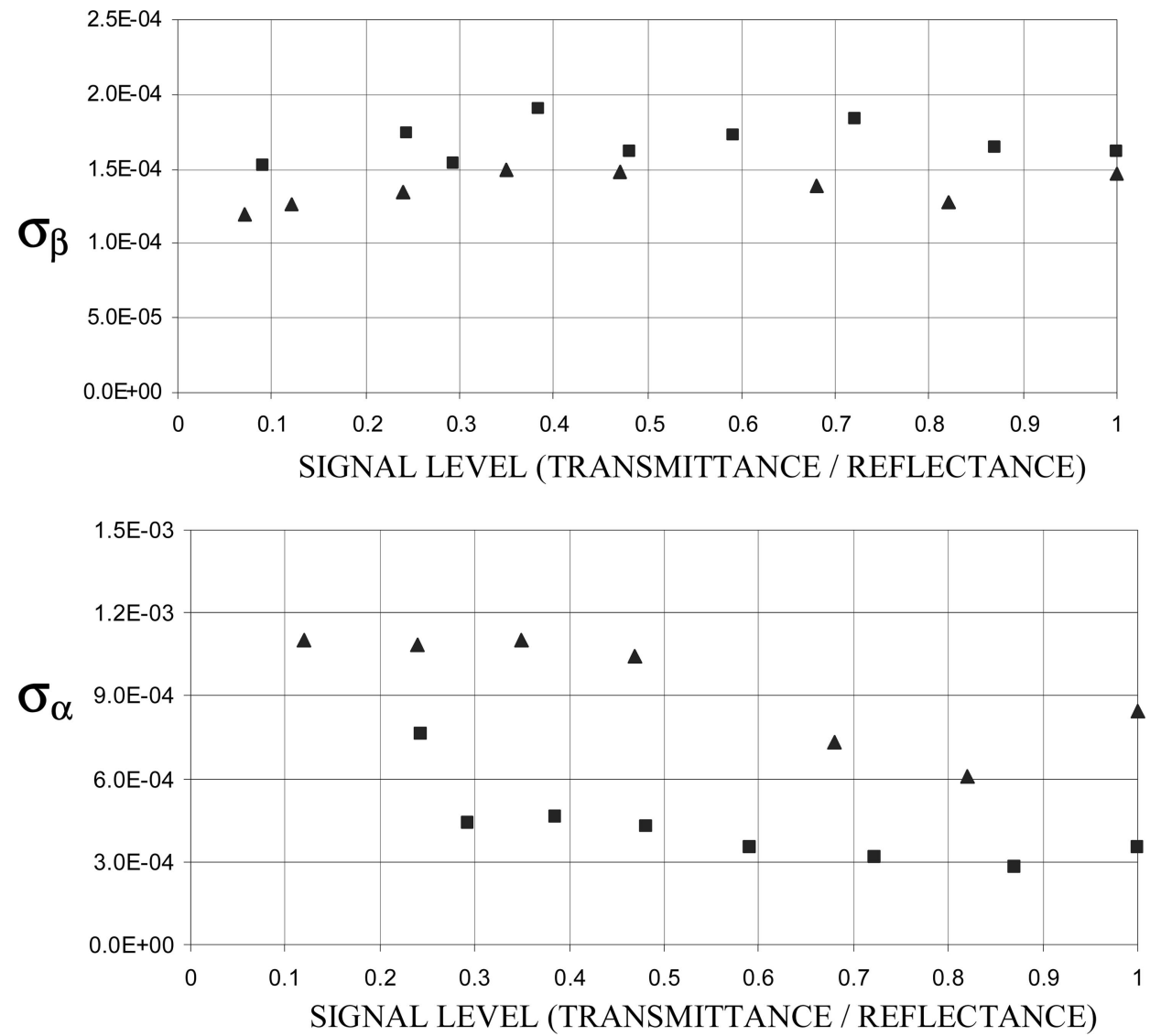

Fig. 2. Experimental values of $\sigma_{\alpha}$ and $\sigma_{\beta}$ as a function of the transmittance (square symbols) and reflectance (triangle symbols) at $600 \mathrm{~nm}$.

In Section 4, we will see the first experimental manufacturing of the thin-film filter using this setup.

\section{Comparison of Theoretical Performances of Three Thickness Control Methods on Three Coating Designs}

\section{A. Choice of Different Test Designs}

The three stacks defined below are used under normal incidence:

1. It is not easy to select designs that are representative of all that can be done using thin-film coat-

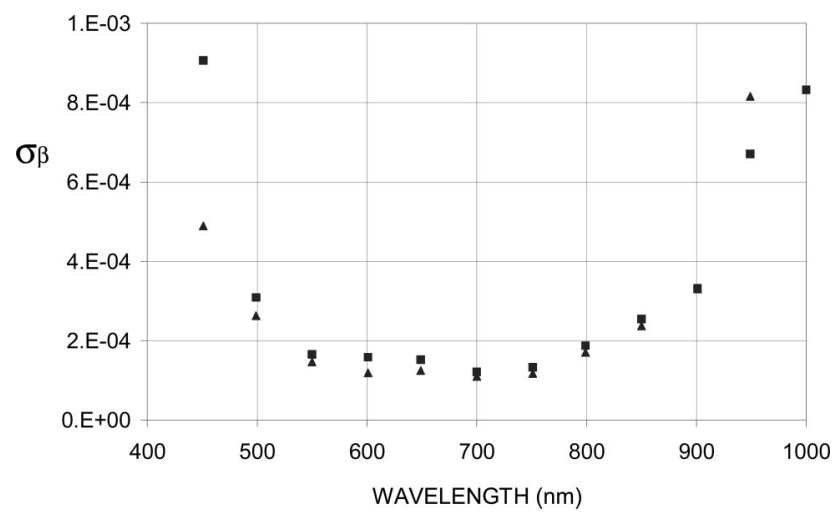

Fig. 3. Evolution of $\sigma_{\beta}$ as a function of the wavelength (transmittance, square symbols; reflectance, triangle symbols). ings. Nevertheless, AR coatings provide a big part of the global optical production. The first filter consists of a six-layer wideband AR component on a glass substrate (refractive index $n s=1.52$ ), with a nominal transmittance above 0.999 between 500 and $800 \mathrm{~nm}$. The corresponding design is given Table 2 , and the transmittance function of the wavelength is plotted in Fig. 4. $H$ and $L$ stand for two dielectric transparent materials of refractive index of 2.35 and 1.45 , respectively. Layers involved in this design are of any thickness varying from 16 to $155 \mathrm{~nm}$.

2 . The second filter is a three-cavity narrow bandpass filter centered at $600 \mathrm{~nm}$, with a spectral full width at half maximum (FWHM) of $9 \mathrm{~nm}$. All thicknesses are quarter-wave, except for the last two thicknesses to ensure an AR behavior at $\sim 600 \mathrm{~nm}$. The design is given in Table 3 , and the transmittance spectra are plotted in Fig. 5. Such a design is a very classical one for narrowband pass filtering components.

Table 1. Experimental Variance of Transmittance and Reflectance Measurement Channels

\begin{tabular}{ccc}
\hline & Transmittance & Reflectance \\
\hline$\sigma_{\alpha}$ & $3.5 \times 10^{-4}$ & $10 \times 10^{-4}$ \\
$\sigma_{\beta}$ & $2 \times 10^{-4}$ & $2 \times 10^{-4}$ \\
$\mathrm{~S} / \mathrm{N}$ & 2500 & 1000 \\
\hline
\end{tabular}


Table 2. Design of the Six-Layer AR Coating

\begin{tabular}{cccc}
\hline Layer & Material & $\begin{array}{c}\text { Thickness } \\
(\mathrm{nm})\end{array}$ & $\begin{array}{c}\text { QWOT }^{a} \text { at } \\
600 \mathrm{~nm}\end{array}$ \\
\hline 1 & $H$ & 16.5 & 0.258 \\
2 & $L$ & 39.9 & 0.385 \\
3 & $H$ & 162.5 & 2.546 \\
4 & $L$ & 21.1 & 0.204 \\
5 & $H$ & 154.8 & 2.426 \\
6 & $L$ & 107.6 & 1.040 \\
\hline
\end{tabular}

${ }^{a}$ Quarter-wave optical thickness.

3. The last design is a 12-layer configuration that is very unstable due to manufacturing errors. The corresponding spectral function is a beam splitter with both theoretical transmittance and reflectance between 0.4996 and $0.5003 \mathrm{~nm}$ in the 500 to $800 \mathrm{~nm}$ range. This design method is a global optimization procedure inspired by [14]. Only an accurate set of thicknesses can provide a satisfying spectral response. The design is given in Table 4 and transmittance versus wavelength is plotted in Fig. 6 .

\section{B. Presentation of Three Thickness Control Methods}

The different monitoring methods have been succinetly described in Subsection 3.A. They correspond to methods experimentally developed in our laboratory.

\section{Quartz Monitoring}

The first method, which is very simple to implement on the deposition chamber, is quartz monitoring. The strategy consists of performing a good calibration of the chamber to assess the evaporated thickness on the substrate holder. This is, of course, an indirect control method, and the expected performances are directly linked to the quality of the calibration for each layer. Other sources of errors are the possibility of having a different evaporation cone shape during deposition or a nonlinearity between the quartz frequency shift and the thickness of the coating.

\section{Optical Monitoring at a Single Wavelength}

The second method consists of analyzing either the transmitted or the reflected flux through the coated substrate at a single wavelength. Most configurations

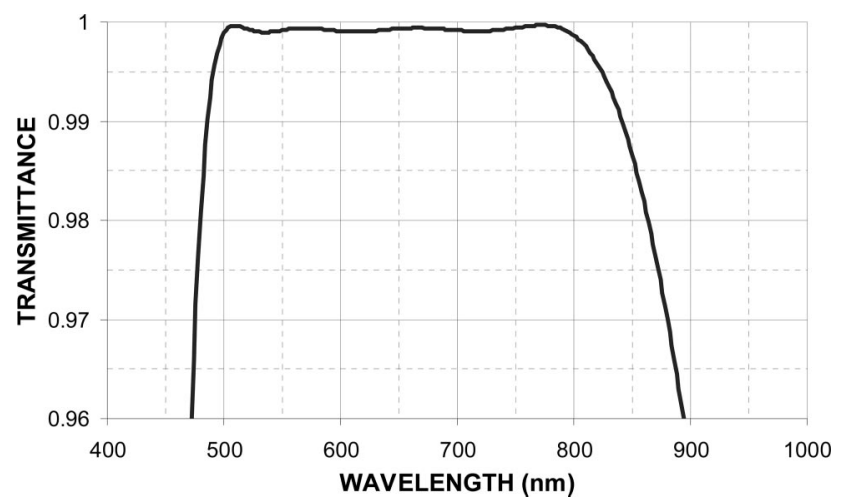

Fig. 4. Theoretical transmittance of the AR coating.
Table 3. Design of the 35-Layer Bandpass Filter

\begin{tabular}{|c|c|c|c|}
\hline Layer & Material & $\begin{array}{l}\text { Thickness } \\
\text { (nm) }\end{array}$ & $\begin{array}{l}\text { QWOT at } \\
600 \mathrm{~nm}\end{array}$ \\
\hline 1 & $H$ & 63.8 & 1 \\
\hline 2 & $L$ & 103.5 & 1 \\
\hline 3 & $H$ & 63.8 & 1 \\
\hline 4 & $L$ & 103.5 & 1 \\
\hline 5 & $H$ & 63.8 & 1 \\
\hline 6 & $L$ & 413.8 & 4 \\
\hline 7 & $H$ & 63.8 & 1 \\
\hline 8 & $L$ & 103.5 & 1 \\
\hline 9 & $H$ & 63.8 & 1 \\
\hline 10 & $L$ & 103.5 & 1 \\
\hline 11 & $H$ & 63.8 & 1 \\
\hline 12 & $L$ & 103.5 & 1 \\
\hline 13 & $H$ & 63.8 & 1 \\
\hline 14 & $L$ & 103.5 & 1 \\
\hline 15 & $H$ & 63.8 & 1 \\
\hline 16 & $L$ & 103.5 & 1 \\
\hline 17 & $H$ & 63.8 & 1 \\
\hline 18 & $L$ & 413.8 & 4 \\
\hline 19 & $H$ & 63.8 & 1 \\
\hline 20 & $L$ & 103.5 & 1 \\
\hline 21 & $H$ & 63.8 & 1 \\
\hline 22 & $L$ & 103.5 & 1 \\
\hline 23 & $H$ & 63.8 & 1 \\
\hline 24 & $L$ & 103.5 & 1 \\
\hline 25 & $H$ & 63.8 & 1 \\
\hline 26 & $L$ & 103.5 & 1 \\
\hline 27 & $H$ & 63.8 & 1 \\
\hline 28 & $L$ & 103.5 & 1 \\
\hline 29 & $H$ & 63.8 & 1 \\
\hline 30 & $L$ & 413.8 & 4 \\
\hline 31 & $H$ & 63.8 & 1 \\
\hline 32 & $L$ & 103.5 & 1 \\
\hline 33 & $H$ & 63.8 & 1 \\
\hline 34 & $L$ & 143.6 & 1.388 \\
\hline 35 & $H$ & 24.9 & 0.390 \\
\hline
\end{tabular}

measure the transmittance, which is the simplest configuration to implement on a vacuum chamber. One possibility is to compare experimental and theoretical values of the transmittance as the criteria for stopping the deposition process. If refractive indexes are well known, with an accurate reproducibility, this technique can be really efficient [14]. The turning point monitoring (TPM) [1] is a variant consisting of choosing the analysis wavelength for which transmittance versus thickness presents a local extremum at the end of the concerned layer. The stopping criterion is applied in the following way: the detection of the experimental extremum is evaluated fitting the measured transmitted signal with a polynomial of degree 2 with an adaptive number of fitting points, depending on the expected signal. When the derivative of the polynomial versus time is equal to 0 , the extremum is found. Such a method is efficient if the deposition rate is stable. A similar approach can be performed with the analysis of reflectance as a function of time at a single wavelength. Note that the measured signal is sequentially acquired if one uses a lateral monitoring 

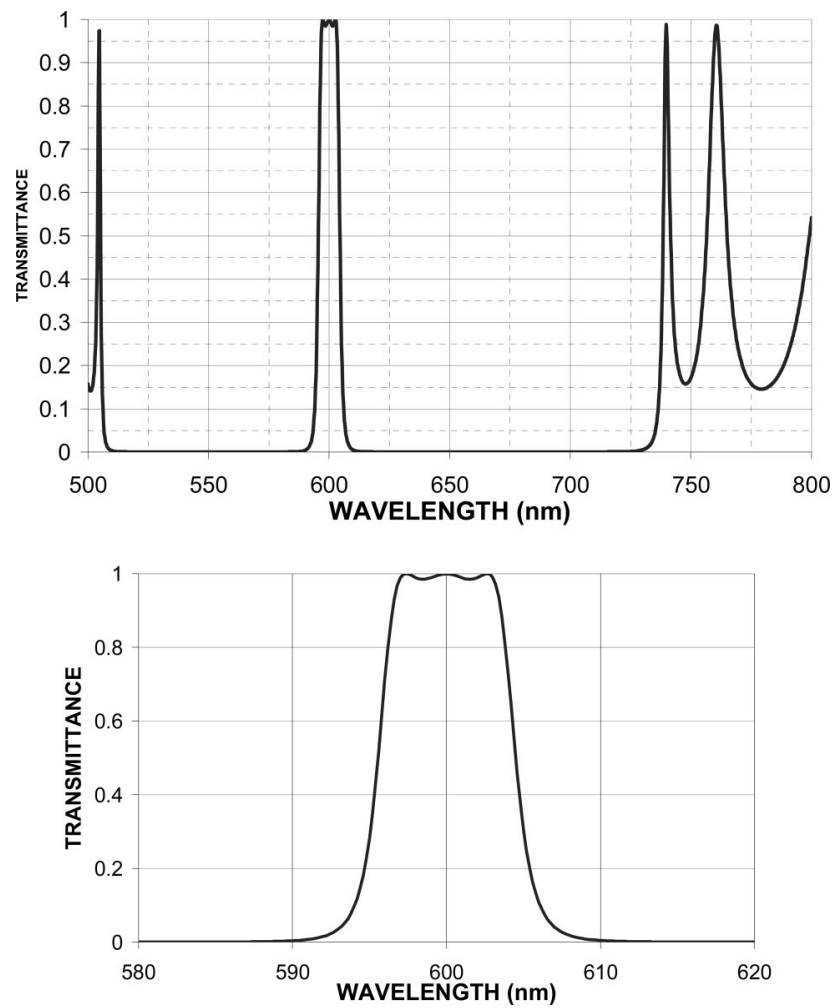

Fig. 5. Theoretical transmittance of the bandpass filter.

system to ensure a good coating uniformity. The signal is then recorded at each turn of the substrate holder.

The efficiency of this optical monitoring has been proven for bandpass filters as deposition errors cause minor deformations at the wavelength of interest in the particular case where all layer optical thicknesses are quarter-waves. Concerning designs with any optical thickness layer, the turning point monitoring method implies the use of different control wavelengths and is generally less robust to manufacturing errors.

Table 4. Design of a $50 \%$ Beam Splitter

\begin{tabular}{cccc}
\hline Layer & Material & $\begin{array}{c}\text { Thickness } \\
(\mathrm{nm})\end{array}$ & $\begin{array}{c}\text { QWOT at } \\
600 \mathrm{~nm}\end{array}$ \\
\hline 1 & $H$ & 17.3 & 0.272 \\
2 & $L$ & 45.0 & 0.435 \\
3 & $H$ & 168.7 & 2.642 \\
4 & $L$ & 28.8 & 0.279 \\
5 & $H$ & 200.1 & 3.134 \\
6 & $L$ & 63.7 & 0.616 \\
7 & $H$ & 73.6 & 1.153 \\
8 & $L$ & 109.0 & 1.054 \\
9 & $H$ & 116.5 & 1.825 \\
10 & $L$ & 20.9 & 0.202 \\
11 & $H$ & 111.6 & 1.748 \\
12 & $L$ & 90.0 & 0.870 \\
\hline
\end{tabular}

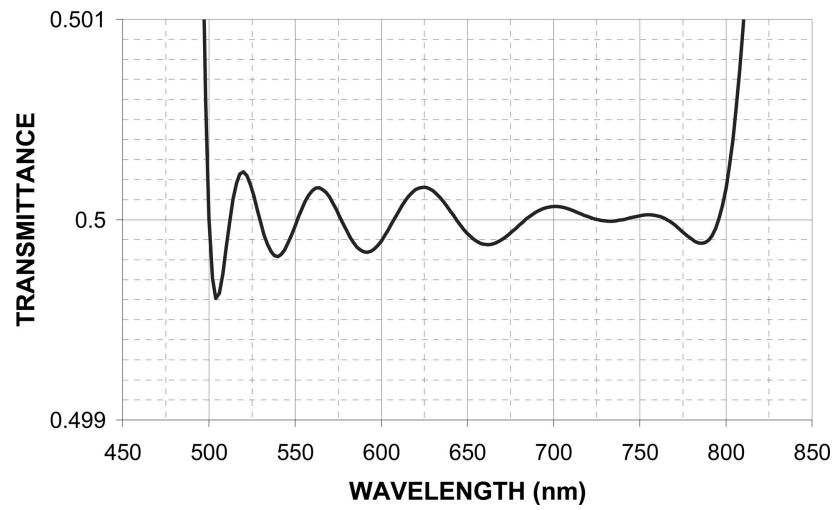

Fig. 6. Theoretical transmittance of the beam splitter.

\section{Optical Broadband Monitoring}

As mentioned in Subsection 3.B.2, this method consists of sequential measurements of transmitted and/or reflected spectra over a wideband spectral interval. At each instant of measurement, the analysis of the spectra provides the most likely thickness of the deposition layer. We are then able to evaluate the deposited thickness as a function of time and compare it with the final theoretical thickness. The stopping criterion of BOM is validated when the theoretical thickness and the real time expected thickness are in coincidence. Note that the sequential acquisition of spectra involves a precision over the deposited thickness that is more or less equal to the deposition rate multiplied by the period of acquisitions.

\section{Influence of Manufacturing Errors on the Different Designs as a Function of the Selected Monitoring Method}

\section{Thickness Errors}

The modeling of thickness errors using quartz crystal monitoring is included in most classical thin-film design software. It consists of replacing each nominal thickness to a new value satisfying a normal law and a specific standard deviation. A well-calibrated quartz monitoring system will induce a standard deviation on thickness deposition errors of approximately $\sigma=2 \mathrm{~nm}$ in the best conditions. This value is quite optimistic considering our quartz crystal monitoring system.

The optical monitoring simulations are more complex. For each layer, we calculate the theoretical signal (transmittance or reflectance) for several growing values of thickness $d$ with a step $\Delta d$ of $0.4 \mathrm{~nm}$, corresponding to the additional deposited thickness during one round of the substrate holder. We simulate measurement data in adding noise [see Eqs. (1) and (2)] to this signal, and we apply the stopping criterion (TPM or BOM) until it is satisfied. Note that we prohibit values of $d$ differing from the theoretical signal by more than $20 \%$.

For TPM modeling, in accordance with the best monitoring systems commercially available, we have chosen a SNR equal to 5000 [14]. The modeling then consists of a standard deviation of transmittance measurements of approximately $\sigma=210^{-4}$ (additive 
noise). If a local extremum at the end of the concerned layer cannot occur at any wavelength, we have considered a quartz monitoring with $\sigma=2 \mathrm{~nm}$. If several wavelengths satisfy the stopping criterion, we have chosen the one corresponding to the most abrupt extremum. For the quarter-wave design, the monitoring wavelength is obviously the central one.

Concerning BOM, the modeling of the noise measurement is the one described in Subsection 2.B by Eq. (1). The chosen parameters are the same as we discussed, except that, for the sake of simplicity, $\sigma_{\beta t}$ is considered to be constant from 400 to $1000 \mathrm{~nm}$ :

$$
\begin{aligned}
& \sigma_{\alpha t}=3.5 \times 10^{-4}, \\
& \sigma_{\beta t}=2 \times 10^{-4} .
\end{aligned}
$$

We have restricted this comparative study to a transmittance setup since most of the commercial systems are available under this restriction. In some case, and specifically for AR coatings, the reflectance setup should be more suitable.

Using such simulation parameters and the stopping criteria defined above, we show in Figs. 7-9 the results of 20 different trials with the three monitor-

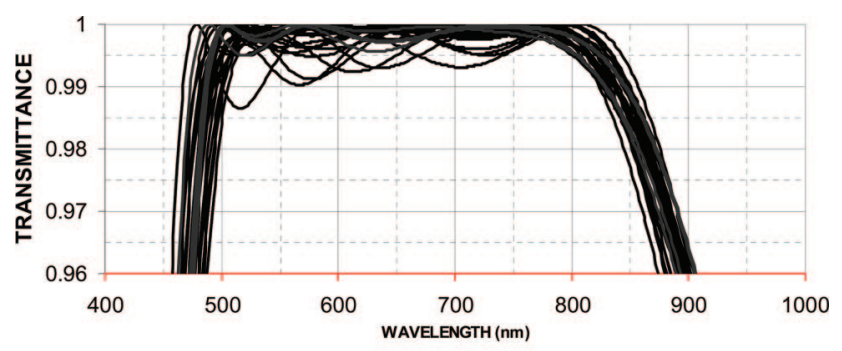

(a)

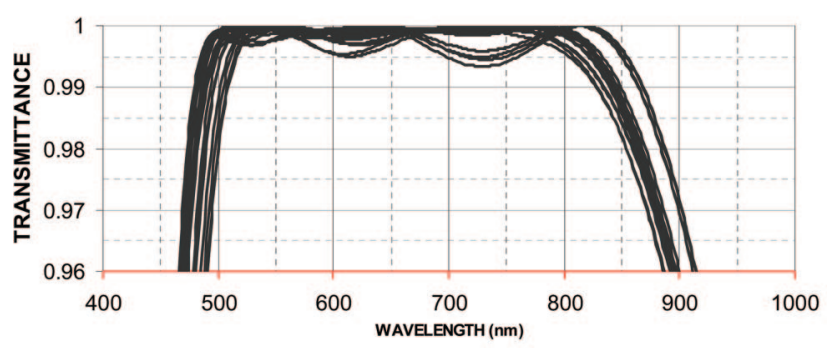

(b)

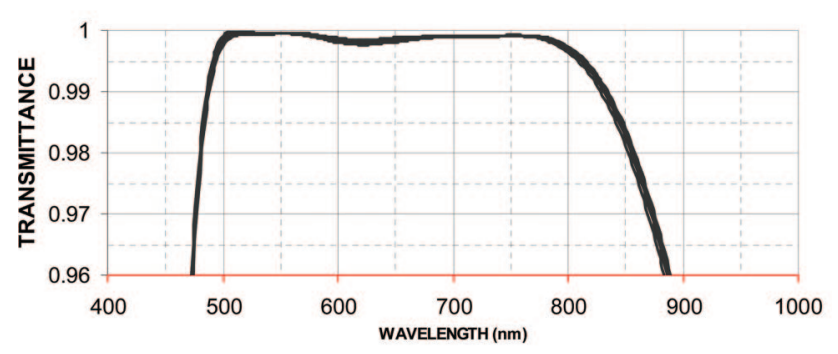

(c)

Fig. 7. (Color online) Calculation of 20 different trials using (a) quartz, (b) TPM, and (c) BOM.

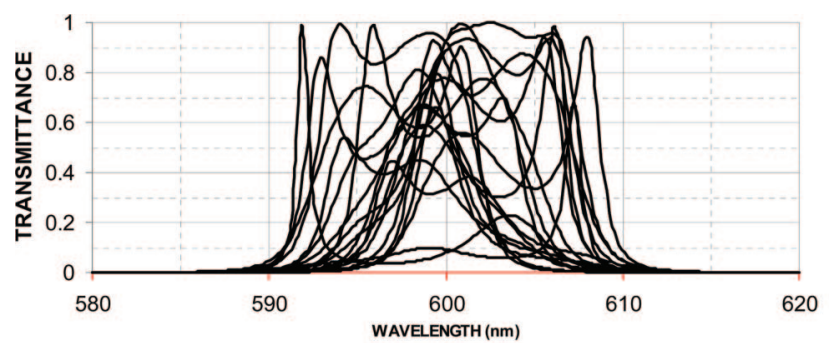

(a)

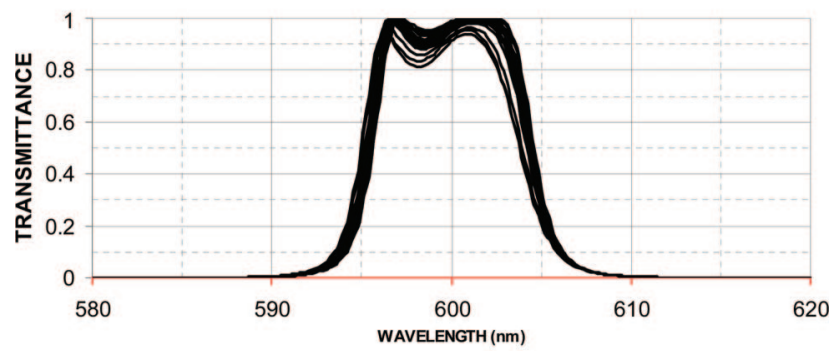

(b)

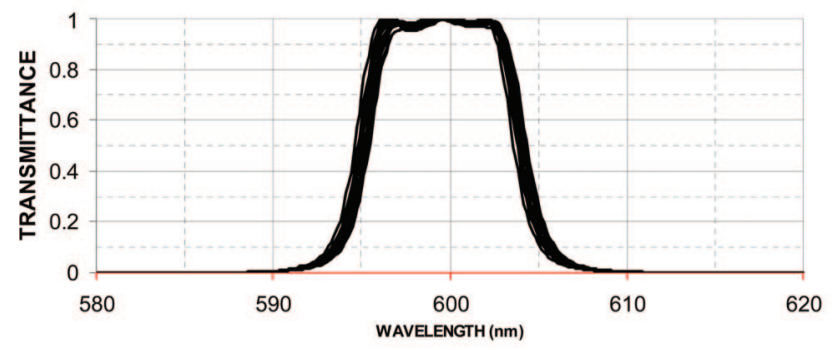

(c)

Fig. 8. (Color online) Calculation of 20 different trials using (a) quartz, (b) TPM, and (c) BOM.

ing methods; (a) stands for quartz monitoring, (b) stands for the TPM method. The set of monitoring wavelengths is given in Table 5. When no monitoring wavelength is found, a quartz monitoring with $\sigma=$ $2 \mathrm{~nm}$ is used; (c) stands for a BOM system using a transmittance over 400-1000 $\mathrm{nm}$.

For the three designs, trials using a quartz monitoring method provide the results with the largest dispersion. As one could expect, the three-cavity bandpass filter cannot be achieved by a simple crystal quartz control. Turning point monitoring provides quite good results, especially concerning the bandpass filter, which is almost a quarter-wave filter. Error compensation is efficiently applied in this configuration. One can note the presence of quasisystematic errors since some curves are superimposed. This is due to the discretization of the possible layer thicknesses with a step of $0.4 \mathrm{~nm}$. BOM seems to be the most efficient method for the three designs. One of the best advantages of this method is that, whereas, TPM is adapted to quarter-wave designs, BOM appears to be efficient in many kinds of designs. Of course, the restriction of the spectral monitoring range from 400 to $1000 \mathrm{~nm}$ to a smaller range increases the dispersion of the transmittance curves. 


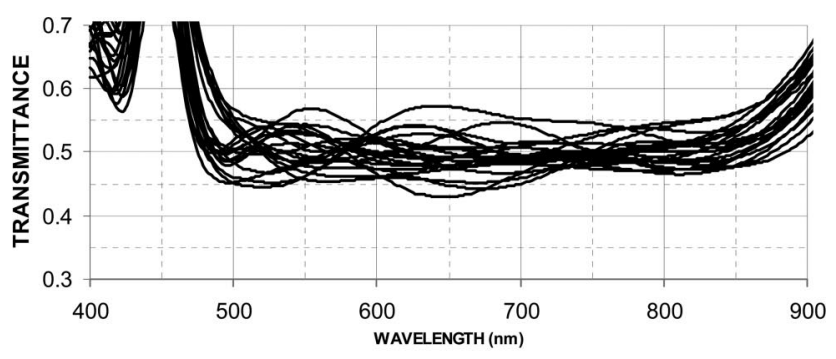

(a)

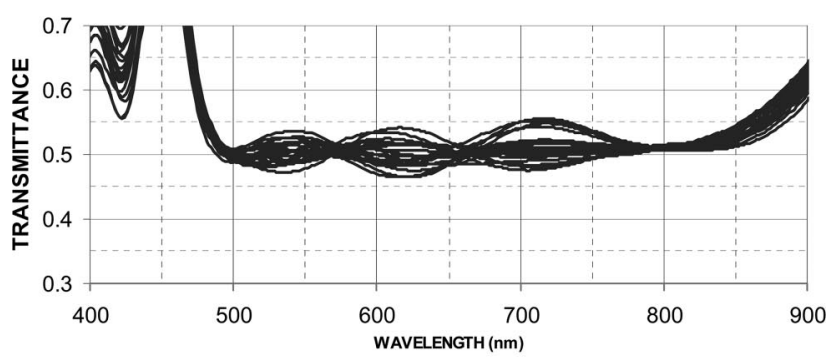

(b)

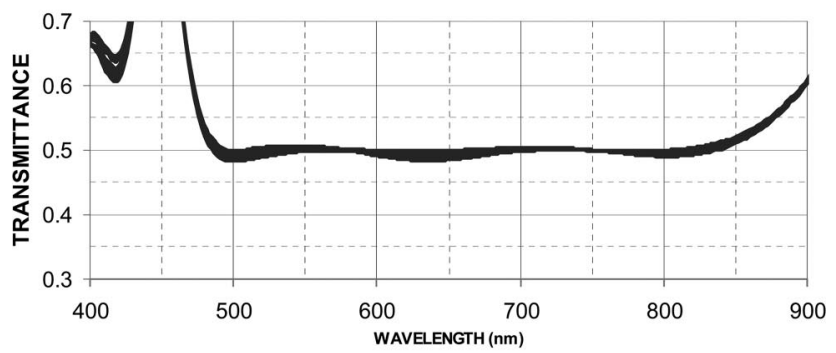

(c)

Fig. 9. Calculation of 20 different trials using (a) quartz, (b) TPM, and (c) BOM.

\section{Refractive Index Errors}

Let us now suppose that the true refractive index of the layer is different from the one expected. We will assume that the refractive index of deposited materials satisfies Eq. (4),

Table 5. Monitoring Wavelengths in Nanometers for TPM Control

\begin{tabular}{cccc}
\hline Layer & AR Coating & Bandpass & Beam Splitter \\
\hline 1 & $Q^{a}$ & 600 & $Q$ \\
2 & 691 & 600 & 771 \\
3 & 793 & 600 & 803 \\
4 & $Q$ & 600 & $Q$ \\
5 & 622 & 600 & 855 \\
6 & 634 & 600 & 484 \\
7 & & 600 & 569 \\
8 & & 600 & 601 \\
9 & & 600 & 568 \\
10 & & 600 & 591 \\
11 & & 600 & 725 \\
12 & & 600 & 658 \\
13 to 32 & & 600 & \\
33 & & 743 & \\
34 & & 981 & \\
\hline
\end{tabular}

${ }^{a}$ Quartz monitoring.

$$
n(\lambda)=a_{0}+a_{1} / \lambda^{2}
$$

where $a_{0}$ satisfies a normal law with a mean value equal to the nominal refractive index value (2.35 for $H$ and 1.45 for $L$ ) and a standard deviation of $\sigma_{a 0}$ $=5 \times 10^{-3} \cdot a_{1}$ satisfies a normal law with a zero mean and a standard deviation of $\sigma_{a 1}=1.6 \times 10^{-3} \mu \mathrm{m}^{2}$. These variations of the refractive index are equivalent to the ones observed with index determination of single layer coatings in our laboratory [15].

It is known [16] that the TPM method is very robust to errors in the specific case of an all quarterwave design, since thickness variations compensate refractive index variation. Using exactly the same stopping criterion as described in Subsection 3.B, we have plotted in Figs. 10(a)-(c) spectra of 20 different trials combining thickness and refractive index errors with (a) quartz monitoring, (b) TPM, and (c) BOM methods on the antireflective coating. Once again, BOM is the most efficient method. Note that we have just presented results for the wideband AR design. For other designs, the conclusion remains the same, but in these cases, the differences between with and without index error are less important (in our index the error configuration is $\sigma_{a 0}=5 \times 10^{-3} / \sigma_{a 1}$ $=1.6 \times 10^{-3} \mu \mathrm{m}^{2}$ ).

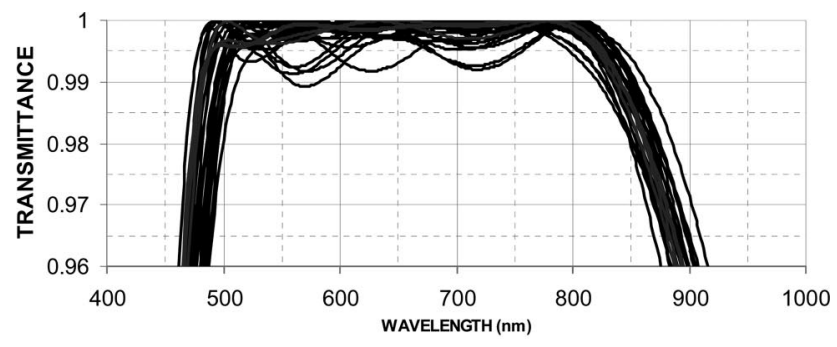

(a)

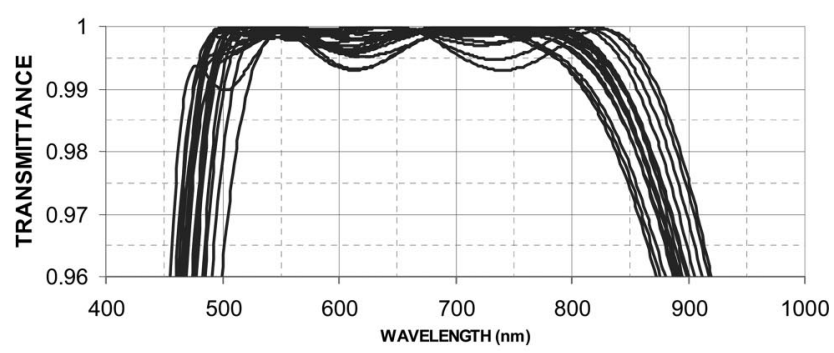

(b)

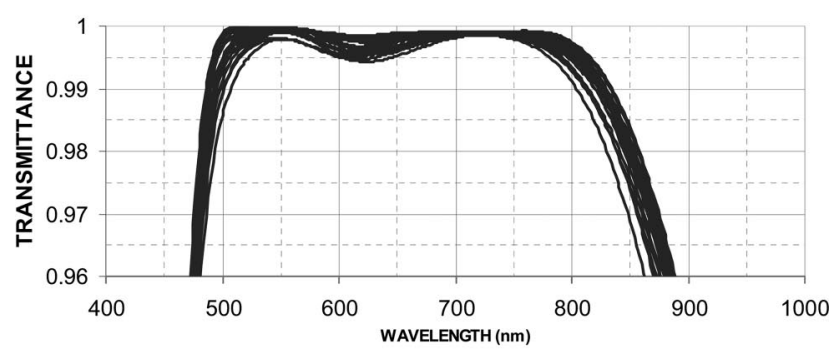

(c)

Fig. 10. Calculation of 20 different trials using (a) quartz, (b) TPM, and (c) BOM. 


\section{Choice of the Adequate Monitoring Method}

The robustness of the deposition control method depends on the expected design. For an all quarterwave design, the TPM is a quite good control method. Nevertheless, BOM performs well for the three different designs, and for many other designs not shown in this paper. Moreover, in the case of the unstable deposition rate, TPM is really inefficient since it does not work if the process unintentionally stops, and the derivative of the flux versus time is null. On the contrary, the BOM system will always provide the expected deposition thickness, and only anticipation on future values of thickness can be eventually wrong in the case of an unstable rate.

\section{Manufacturing of a Thin-Film Filter Controlled with Broadband Optical Monitoring}

To test the performances of our BOM system, we have designed specific $\mathrm{Ta}_{2} \mathrm{O}_{5} / \mathrm{SiO}_{2}$ filters, which are aimed at being used as cavity mirrors for a coherent antiStokes Raman-scattering (CARS) experiment in the team of the Mosaic of Institut Fresnel. The required specifications are the following: under normal incidence, for wavelengths in the range of 570 to $675 \mathrm{~nm}$, the reflectance should be $\sim 0.75 \pm 0.02$, and from 710 to $920 \mathrm{~nm}$, the reflectance should not exceed 0.04 . Such specifications led us to synthesize the filter given in Table 6. Single layer classical characterizations give us the refractive index of the two materials as

$$
\begin{aligned}
n_{\mathrm{Ta}_{2} \mathrm{O}_{5}}(\lambda) & =2.15+2.1510^{-2} / \lambda^{2}+1.4610^{-3} / \lambda^{4}, \\
k_{\mathrm{Ta}_{2} \mathrm{O}_{5}} & =10^{-3} \\
n_{\mathrm{SiO}_{2}}(\lambda) & =1.45+2.4410^{-3} / \lambda^{2} \\
k_{\mathrm{SiO}_{2}} & =0
\end{aligned}
$$

Table 6. Design of a Long-Pass Filter

\begin{tabular}{cccc}
\hline Layer & Material & $\begin{array}{c}\text { Thickness } \\
(\mathrm{nm})\end{array}$ & $\begin{array}{c}\text { QWOT at } \\
700 \mathrm{~nm}\end{array}$ \\
\hline 1 & $H$ & 106.6 & 1.337 \\
2 & $L$ & 272.5 & 2.273 \\
3 & $H$ & 88.6 & 1.112 \\
4 & $L$ & 109.1 & 0.910 \\
5 & $H$ & 205.8 & 2.582 \\
6 & $L$ & 119.3 & 0.995 \\
7 & $H$ & 64.3 & 0.807 \\
8 & $L$ & 298.2 & 2.488 \\
9 & $H$ & 314.0 & 3.939 \\
10 & $L$ & 36.1 & 0.301 \\
11 & $H$ & 373.8 & 4.689 \\
12 & $L$ & 200.5 & 1.673 \\
13 & $H$ & 126.8 & 1.591 \\
14 & $L$ & 212.2 & 1.770 \\
15 & $H$ & 110.5 & 1.386 \\
16 & $L$ & 209.3 & 1.746 \\
17 & $H$ & 127.6 & 1.601 \\
18 & $L$ & 95.5 & 0.796 \\
\hline
\end{tabular}

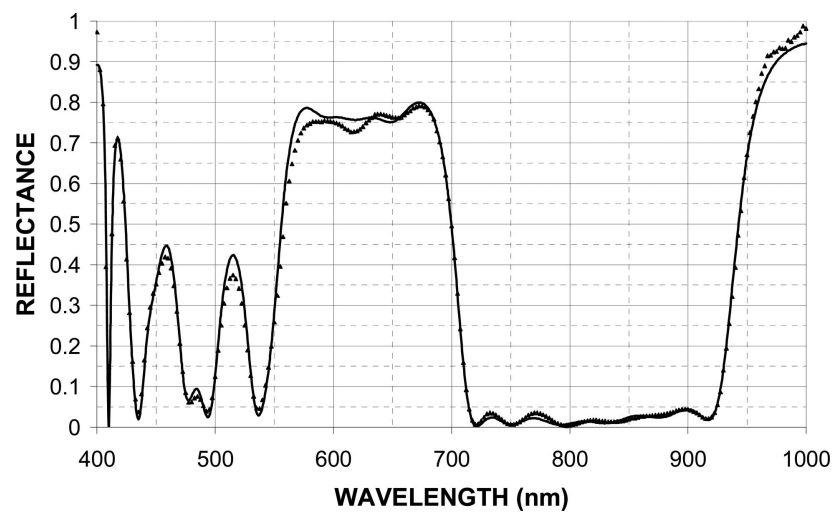

Fig. 11. Manufacturing of a long-pass filter: theoretical data (full curves) and experimental data at the end of the deposition process (dashed curves).

where $n$ stands for the real part and $k$ stands for the imaginary part of the refractive index. The substrate is fused silica with refractive index $n s$ given by a Sellmeier formula [17]. $n_{s}$ is approximately 1.46 at $550 \mathrm{~nm}$.

The strategy is the one given in Subsection 3.B: at each round of the substrate holder, the system analyzes both reflectance and transmittance and estimates the deposition thickness. When the theoretical threshold is passed, the deposition process is stopped. Figure 11 provides the expected and measured reflectance at the end of the deposition process. The residual error $(\mathrm{RE})$ or root mean square error between the theoretical and measured transmittances, given by Eq. (7) is equal to 0.017:

$$
\begin{aligned}
\mathrm{RE} & =\sqrt{\frac{1}{N_{\lambda}} \sum_{\lambda \in D}\left(R_{\text {meas }}(\lambda)-R_{\mathrm{th}}(\lambda)\right)^{2}}, \\
D & =[570-675 \mathrm{~nm}] U[710-920 \mathrm{~nm}] .
\end{aligned}
$$

This first experimental result is rather encouraging and is consistent with simulation results considering both thicknesses and refractive index errors in Section 3.

\section{Conclusion}

We have developed a broadband optical monitoring (BOM) for thin-film filter manufacturing working in both transmittance and reflectance. The two spectral responses are recorded at each turn of the substrate holder over a 400-1000 $\mathrm{nm}$ range, and the deposited thickness is estimated. For a transmittance (respectively, reflectance) equal to 1 , the signal-to-noise ratio is $\sim 2500$ (respectively, 1000), and the behavior of the real-time measurements has been modeled with a combination of normal laws. We have then evaluated the theoretical robustness of BOM compared with turning point monitoring and quartz monitoring. In most cases, BOM is the most efficient method as long as the spectral range for the thickness determination is wide. The use of such a monitoring system analyzing, simultaneously, both transmit- 
tance and reflectance should also enable in situ real determination of the real and imaginary parts of refractive indexes. A real-time re-engineering process of reoptimizing the coating design after each layer is then conceivable. Our future developments will be reported in this manner.

\section{References}

1. H. A. Macleod, "Monitoring of optical coatings," Appl. Opt. 20, 82-89 (1981).

2. C. Buzeal and K. Robbie, "State of the art in thin film thickness and deposition rate monitoring sensors," Rep. Prog. Phys. 68, 385-409 (2005).

3. H. D. Polster, "Symmetrical all-dielectric interference filter," J. Opt. Soc. Am. 42, 21-24 (1952).

4. http://www.leyboldoptics.com/en/oms5000.html.

5. S. N. Jasperson, D. K. Burge, and R. C. O'Handley, "A modulated ellipsometer for studying thin film optical properties and surface dynamics," Surf. Sci. 37, 548-558 (1973).

6. M. Cathelinaud, F. Lemarquis, and C. Amra, "Index determination of opaque and semitransparent metallic films. Application to light absorber," Appl. Opt. 41, 2546-2554 (2002).

7. D. Ristau, T. Gross, and M. Lappschies, "Optical broadband monitoring of conventional and ion process," in Optical Interference Coatings, OSA Technical Digest Series (Optical Society of America, 2004), paper TuE1.

8. D. Ristau, H. Ehlers, T. Gross, and M. Lappschies, "Optical broadband monitoring of conventional and ion processes," Appl. Opt. 45, 1495-1501 (2006).

9. X. Q. Hu, Y. M. Chen, and J. F. Tang, "Apparatus for wideband monitoring of optical coatings and its uses," Appl. Opt. 28, 2886-2888 (1989).

10. S. Wilbrandt, N. Kaiser, and O. Stenzel, "In situ broadband monitoring of heterogeneous optical coatings," Thin Solid Films 502, 153-157 (2006).

11. S. Wilbrandt, R. Leitel, D. Gabler, O. Stenzel, and N. Kaiser, "In situ broadband monitoring and characterization of optical coatings," in Optical Interference Coatings, OSA Technical Digest Series (Optical Society of America, 2004), paper TuE6.

12. A. V. Tikhonravov, M. K. Trubetskov, and T. Amotchkina, "Investigation of the effect of accumulation of thickness errors in optical coating production using broadband optical monitoring," Appl. Opt. 45, 7026-7034 (2006).

13. K. B. Walsh, J. A. Guthrie, and J. W. Burney, "Application of commercially available, low cost, miniaturized NIR spectrometers to the assessment of the sugar content of intact fruit," Aust. J. Plant. Physiol. 27, 1175-1186 (2000).

14. A. Zoeller, "Substantial progress in optical monitoring by intermittent measurement technique," in Advances in Optical Thin Films II, Proc. SPIE 5963, 59630D (2005).

15. F. Lemarchand, C. Deumie, M. Zerrad, L. Abel-Tiberini, B. Bertussi, B. Lazaridès, M. Cathelinaud, M. Lequime, and C. Amra, "Optical characterization of an unknown single layer: Institut Fresnel contribution to OIC 2004 measurement problem," in Optical Interference Coatings (Optical Society of America, 2006), pp. 1312-1318, paper WE9.

16. H. A. Macleod, Thin-Film Optical Filters, 2nd ed. (Hilger, 1986).

17. Fused silica refractive index data: http://en.wikipedia.org/wiki/ Fused_quartz. 